\title{
Mobile Agent-Based Load Monitoring System for the Safety Web Server Environment
}

\author{
H.J. Park ${ }^{1}$, K.J. Jyung ${ }^{2}$, and S.S. Kim ${ }^{3}$ \\ ${ }^{1}$ School of Computer Information and Communication Engineering, \\ Sangji University, Woosandong, Wonjusi, Kangwondo, Korea \\ hjpak1@sangji.ac.kr \\ ${ }^{2}$ System Technology Laboratory, Dacom Corporation, Seoul, Korea \\ jkjin@origio.net \\ ${ }^{3}$ School of Information and Communication Engineering, \\ Halla University, San 66, Heungup-Li, Heungup-myon, Wonjusi, Kangwondo, Korea \\ sskim@halla.ac.kr
}

\begin{abstract}
The importance of the server is growing with the growth of the internet and the load on the server has rapidly grown with the increase in the number of web server user. Load monitoring has become an important technical aspect in the performance of the server for these reasons. It has proven to be more efficient to use a java mobile agent that operates by moving between the systems for load monitoring of a web server rather than using the existing client-server paradigm. A mobile agent moves around the web servers to measure the load and sends the load information to a single node.
\end{abstract}

\section{Introduction}

The importance of the web server that provides web service to users all around the world has grown rapidly with the growth of the internet. Mirroring method has also been applied on the web servers for load balancing but has proven inefficient due to the difficulty in managing the performance of the web servers in remote location. Load monitoring technique on many distributed web servers is an important aspect in the management of web server performance.

A web server must always provide the user with the appropriate service and speed of the service is the most important aspect. If the load on the web server increases by the increase in the number of user, size of data, number of process or the use of memory, the server would not be able to provide the user with the appropriate service and would cause a waste of money put into developing the web server[1]. The load monitoring system must continuously check if the web server is operating properly, and must apply the optimization process to restrain the number of user or size of data for the server to operate efficiently. Accurate load information can be applied to efficiently distribute the load on a server to another mirroring web server and the web server monitoring technique can be applied to promptly detect and resolve an error that has occurred within the system. The system administration condition can be predicted based on the historical data stored in the DB and this data can also be used as the base information for analysing and expanding the system. 
This paper develops a load monitoring system using a java mobile agent that can efficiently monitor the load on the web server and resolve the problems that occurred on the systems based on the client-server paradigm.

This paper consists of the following parts. Chapter 2 deals with the related research, chapter 3 with the design and implementation of the monitoring system using a mobile agent and chapter 4 concludes the paper by analyzing the implemented system.

\section{Related Research}

\subsection{Load Monitoring in the Client-Server Paradigm}

The client-server paradigm has been the base for most web-server load monitoring systems. The client-server paradigm is a centralized method in the distributed computing environment and must install a program that measures the load of the web server on each server and send the load information on a demand from the client[2]. Therefore, it is impossible for a user to change the monitoring method on a single node and to manage web servers that are remotely located. Also, the monitoring program being operated on the web server may become a load on the server providing the web service and the message shared between the servers increases the network traffic. A network connection must be maintained between the client and the web server and therefore becomes hard for the web server to adapt to the change in the environment[3][4]. The mobile agent is applied to resolve such problems of the clientserver program by gathering information by moving through the servers.

\subsection{Java Mobile Agent}

The java mobile agent is a program that can be operated by moving itself to another host on the network. The mobile agent can transfer its state information and code from its initial environment to the system it is to be operated on. The state information is the attribute value used to determine the job to be processed by the agent and the code is a class code needed for the agent's operation. The mobile agent can operate efficiently in an environment where systems connected through the network have a low bandwidth and high delay time. The agents can also share their resource through communicating with each other and returns to the system it was created or selfdestructs after the finishing its job. The use of a mobile agent provides a easier and more efficient programming paradigm than the client-server paradigm in constructing an application in the distributed computing environment[5].

The agent takes an efficient approach of moving around the system through a connected network for gathering data and utilizing resource instead of requesting other systems and waiting for a reply. Network connection is only required when the mobile agent is actually moving through the system and the user does not need to take the location of the operation taking place or the communication channel into account. This enables the development, testing and locating of the distributed application to be operated more efficiently (Fig. 1). 


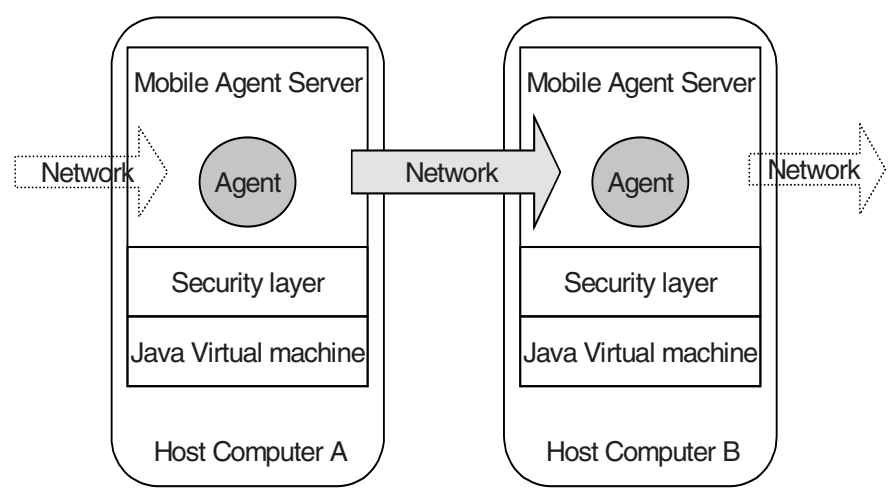

Fig. 1. Java mobile agent

\subsection{Advantage of the Java Mobile Agent}

Following are the advantages of using a Java mobile agent[6].

- Reduced load on the network: The network traffic increases on a distributed system environment where a lot of interaction takes place between the systems, whereas a mobile agent only requires a small amount of data to be transferred within the system.

- Resolve Network Delay: Network delay occurs on a real-time system on a process through the network. However, a mobile agent can directly process the job issued by the central manager by moving itself.

- Asynchronous and Free operation: A mobile agent only requires a network connection on a movement and operates freely and asynchronously within the system it has moved to.

- Protocol Encapsulation: An I/O related protocol must be formed to exchange data in a distributed system environment, whereas a mobile agent uses an independent protocol to move to a remote host.

- Dynamic Adaptation: Detect the operating environment and automatically respond to the change.

- Heterogeneous: Network computing through the internet contains a heterogeneous nature.

- Fault Tolerance: Able to construct a fault-tolerant distributed system even when a problem has occurred.

\section{Web Server Load Monitoring System Using a Mobile Agent}

This chapter discuss about the advantages of monitoring using a mobile agent and the design and implementation of a web server load monitoring system. 


\subsection{Mobile Agent Based Monitoring}

Using a mobile agent with the above characteristics for monitoring the load on a web server is far more efficient than implementing the existing client-server paradigm.

All the load data can be gathered into a single system without installing a program on each web server to be monitored by using a mobile agent. The execution code of the mobile agent moves to a web server through the network to internally measure the load and sends the result to the monitoring server before moving onto another web server. The mobile agent only contains the code that is crucial to measuring the load to become a lightweight program and requires only a small amount of data upon a network transaction. It also puts only a little extra load on the web server by only taking up little room for execution upon a movement. A large-scale log file is required for measuring the load on the web server. However, by using a mobile agent, the agent will carry the log file itself instead of transmitting it through the network for a quicker analysis of the load data.

The mobile agent is an independent protocol that moves through the ATP(Agent Transfer Protocol). This enables the operation of the agent to proceed regardless of the physical location of the web server to be monitored and is not restricted by the number of web servers. The load of mirroring web servers that are physically distributed can be monitored from a single node and the resulting data can be utilized for the load balancing technique of a mirroring web server. Therefore, it can be concluded that the monitoring method using a mobile agent is more efficient than the existing client-server program.

\subsection{Design}

A distributed computing method using a mobile agent is required for an efficient load monitoring on web servers that are remotely located on the internet. The system consists of web servers to be monitored, and a monitoring system used to monitor the load information of the web servers. Each system contains a MAS (Mobile Agent Server). MAS (Mobile Agent Server) provides each host with an environment for the mobile agent to be executed and manages the creation, movement and extinction of the mobile agent. The agent is designed by combining the itinerary pattern and the master-slave pattern related to the operation. The Master agent is executed on the MAS of the monitoring system, and creates the Slave agent that will move to the web server. The Slave agent moves to the first web server to analyze the log file, measure the load on the system and sends the load information to the Master agent. The Slave agent then moves to another agent to perform the same job after transmitting the load information to the Master slave.

The master agent resides in the monitoring system to provide the GUI function, display the web server's load information gathered from the Slave agents to the users and periodically create a new Slave agent. These functions of the mobile agent are shown in Fig. 2. 


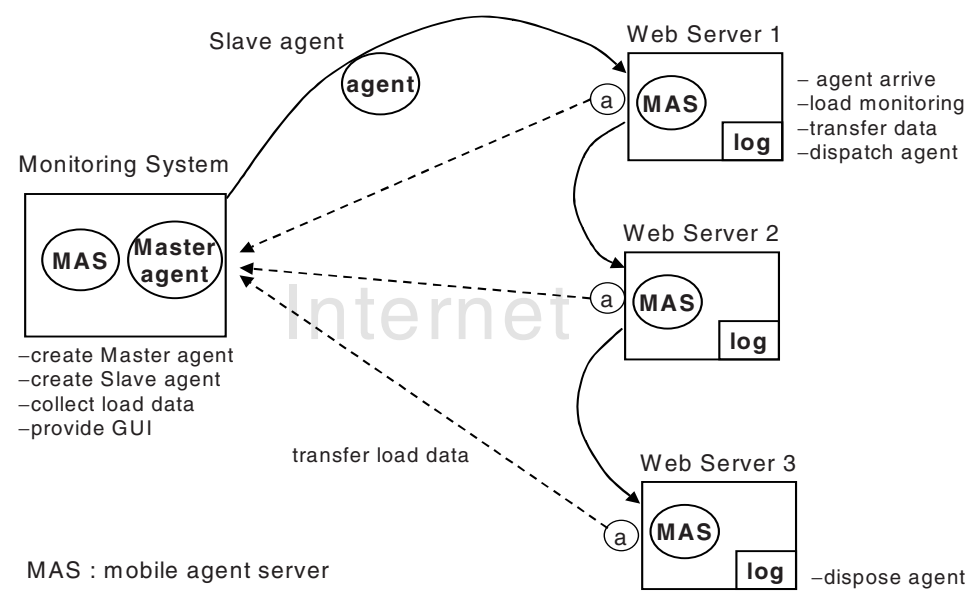

Fig. 2. Function of master and slave agent using itinerary pattern

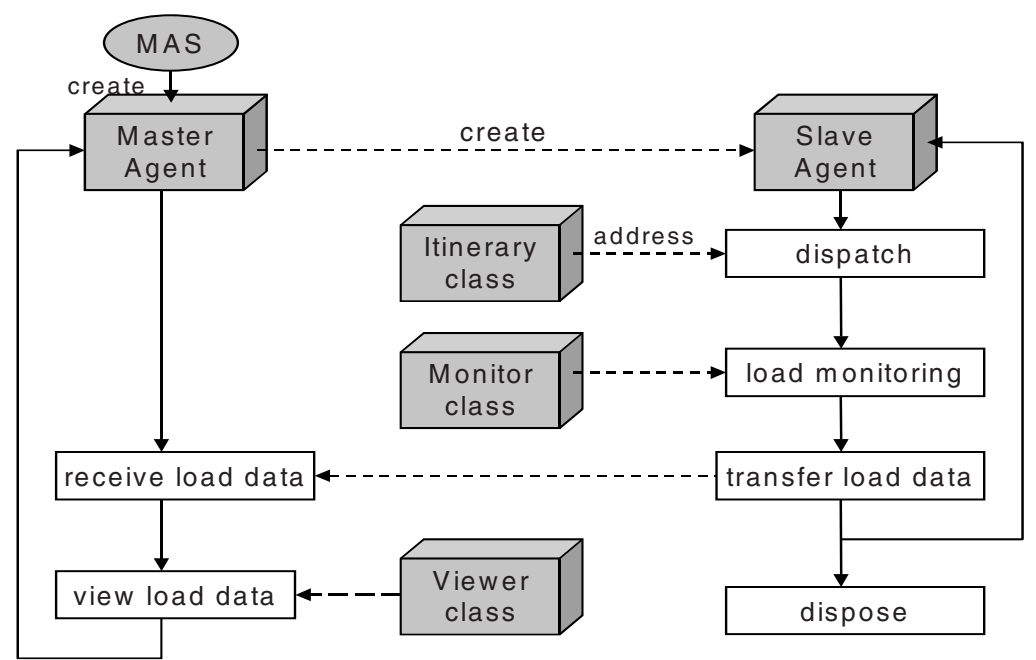

Fig. 3. Classes of load monitoring system

\subsection{Implementation}

The web server monitoring system using a java mobile agent consists of a master agent and a slave agent. The master agent displays the load information obtained from the slave agent through the viewer class(GUI function) and periodically creates a new slave agent for monitoring. The slave agent created by the master agent moves itself to the web server. The address of the web server the slave agent is to move to is provided by the itinerary class. The itinerary class contains the address of the web servers to be monitored and is transmitted upon the creation of the slave agent by the master agent. The itinerary class delivers the agent to the next address and provides 
the function for moving on to the following server if a problem occurs at the web server (Fig. 3).

The slave agent that moved to the web server analyses the log data of the web server and measures the load on the system itself. It moves to another web server after transmitting all the load data to the Master slave, and self-terminates if there is no other job left to process. The monitor class measures the number of current users, current transmission rate, number of currently occurred errors, average number of jobs processed in $1 \cdot 5 \cdot 15$ minutes interval, size of the swap memory, size of free memory, rate of disc usage, amount of memory left in the disc and the amount of memory left to be used in the disc. The load information of the web server is displayed in the monitoring system as shown in Fig. 4.

\begin{tabular}{|c|c|c|c|c|c|c|c|c|c|c|c|c|c|}
\hline \multicolumn{13}{|c|}{ Web Server Load } & \multirow[t]{2}{*}{$-\square^{2}$} \\
\hline \multicolumn{13}{|c|}{ [ Web Server Load] } & \\
\hline server & ping & user & $\begin{array}{l}\text { transfer } \\
\text { volume }\end{array}$ & $\begin{array}{l}\text { err } \\
\text { or }\end{array}$ & $\begin{array}{l}\text { Imin } \\
\text { load }\end{array}$ & $\begin{array}{l}5 \mathrm{~min} \\
\text { load }\end{array}$ & $\begin{array}{l}15 \mathrm{~m} \\
\text { load }\end{array}$ & $\begin{array}{c}\text { swap } \\
\text { memory }\end{array}$ & $\begin{array}{c}\text { free } \\
\text { memory }\end{array}$ & \begin{tabular}{|l} 
disk \\
util
\end{tabular} & $\begin{array}{l}\text { used } \\
\text { disk }\end{array}$ & $\begin{array}{l}\text { avail } \\
\text { disk }\end{array}$ & time \\
\hline turbo & 1 & 1 & 58731 & $\mathbf{0}$ & 0.05 & 0.47 & 0.79 & 119848 & 1376 & 0.0 & 1175611 & 2551335 & 11:20:07 \\
\hline orchestra & 2 & 1 & 76588 & 1 & 0.12 & 0.15 & 0.14 & 315936 & 16272 & 0.0 & 2838818 & 2528952 & 11:19:50 \\
\hline \multirow{2}{*}{ opera } & 1 & 3 & 437149 & 2 & 0.04 & 0.04 & 0.05 & 886704 & 181592 & 0.0 & 10727548 & 15618537 & 11:19:51 \\
\hline & ms & & bytes & & jobs & jobs & jobs & Kbytes & Kbytes & $\%$ & bytes & bytes & \\
\hline \multicolumn{14}{|c|}{ Close } \\
\hline
\end{tabular}

Fig. 4. Load information display of web servers

\section{Conclusion}

The size of the program operated in each web server is as follows. The program in the Slave agent takes up 3.2KB, in the Monitor class takes up $4.3 \mathrm{~KB}$, and the program in the Itinerary class takes up $2 \mathrm{~KB}$ which adds up to a total size of $9.5 \mathrm{~KB}$. $9.5 \mathrm{~KB}$ of class is executed during the measurement and is destroyed after the process which is the main reason this system fares better than the client-server program in which memory space is taken up the whole time. The whole class is only transmitted once upon the first agent movement and only the Slave agent is transmitted after the first transmission which results in $9.5 \mathrm{~KB}$ of data only being transmitted once and only $3.2 \mathrm{~KB}$ of data being transmitted after the initial transmission. This reduces the network traffic.

The purpose of this paper is on effectively monitoring the load on the web servers physically distributed on the internet using a java mobile agent. By using the java mobile agent, it becomes possible to monitor more than one web servers from a single node without installing any other program and enables the user to modify the monitoring method. It provides a new mechanism that was not provided in the existing client-server paradigm. 


\section{References}

1. Web Server Monitoring, http://www. freshtech.com

2. M. Caprini, R. Nacach, Z. Qian, "Java Mobile Agent for monitoring task : evaluation report", http: //atddoc.cern.ch/Atlas/Notes/078/Note078-1.html, 1998

3. MARS(Monitoring Application for Resources and Servers), http: / / www . altara.org/mars.html

4. E. Anderson, D. Patterson, "Extensible, Scalable Monitoring for Clusters of Computerts", Proceedings of 1997 LISA Conference, 1997

5. R. Gray, D. Kotz, S. Nog, D. Rus, G. Cybenko, "Mobile agents for mobile computing", Technical Report PCS-TR96-285, 1996

6. D. B. Lange, Mitsuru Oshima, Programming and Deploying Java Mobile Agents with Aglets, Addison Wesley press, 1998 